\title{
¿ES EL SCORE SOFA UN PREDICTOR ADECUADO PARA EVALUAR LA MORTALIDAD MATERNA?
}

\author{
IS SOFA SCORE AN ADECUATE PREDICTOR FOR ASSESMENT OF \\ MATERNAL MORTALITY?
}

Joseph Alburqueque Melgarejo1,a, Juan Carlos Roque Quezada ${ }^{3, c}$

\section{Estimado señor editor:}

Luego de haber leído con interés el artículo "Utilidad del score SOFA en la predicción de muerte materna en la UCI materna del INMP" por De la Peña Meniz Walter et al. publicado en el Volumen 9 número 3 de la Revista Peruana de Investigación Materno Perinatal, el cual consideramos un estudio de suma relevancia en el campo de la Gineco-Obstetricia y la Medicina Intensivista; y teniendo en cuenta la alta tasa de mortalidad materna en el Perú; quisiéramos mencionar algunos aspectos que deberían considerarse para posteriores estudios.

Actualmente no existe un score específico para predecir la mortalidad materna en pacientes obstétricas en la $\mathrm{UCl}$. Sin embargo, a pesar de que no hay muchos estudios al respecto, el score SOFA es el que ha presentado los mejores resultados en contraste con otros scores para valorar mortalidad. Esto puede verse ilustrado en el hecho de que otros scores como el APACHE II o el SAPS II sobreestiman la mortalidad materna, este no es el caso del score SOFA ${ }^{1-3}$.

Por otro lado, es de relevancia señalar las características del score SOFA que hacen que sea un score confiable para la estimación de la variable mortalidad materna, en comparación con otros scores de mortalidad. Una de ellas reside en el hecho de las variables que evalúa el score SOFA que, a diferencia de otros scores, no presentan modificaciones considerables en el estado fisiológico del embarazo. Sin embargo, el score SOFA también algunas variables que si podrían modificarse con este estado fisiológico, estas son las siguientes: presión arterial media, niveles de creatinina sérica y conteo de plaquetas. Además, el score SOFA considera variables que pueden ser evaluadas en estancias de escasos recursos, a diferencia de otros scores ${ }^{1,4}$.

Aunque existen varios subtipos de score SOFA, que tienen diferentes utilidades. En el presente estudio se valoró el score SOFA en la admisión y a las $48 \mathrm{~h}$ del ingreso. Con la presencia de estas dos mediciones hubiera sido interesante el cálculo del delta SOFA, el cual se obtiene del cambio entre el score SOFA total en la admisión y en un punto definido en el tiempo. Esta sugerencia se sustenta en el hecho de que el delta SOFA tiene más confiabilidad para la evaluación de mortalidad en los ensayos clínicos ${ }^{4,5}$.Es importante también mencionar que la medida del score SOFA debe ser adecuada, puesto que errores al momento de su medición pueden conllevar a variabilidad en los resultados entre diferentes autores ${ }^{4}$.

Finalmente, mencionar que el score SOFA es un instrumento útil para evaluar la mortalidad materna, dada su superioridad en relación a otros scores previamente mencionados.

Contribuciones de autoría: los autores han participado en la concepción y aprobación de la versión final del manuscrito.

Fuentes de financiamiento: autofinanciado.

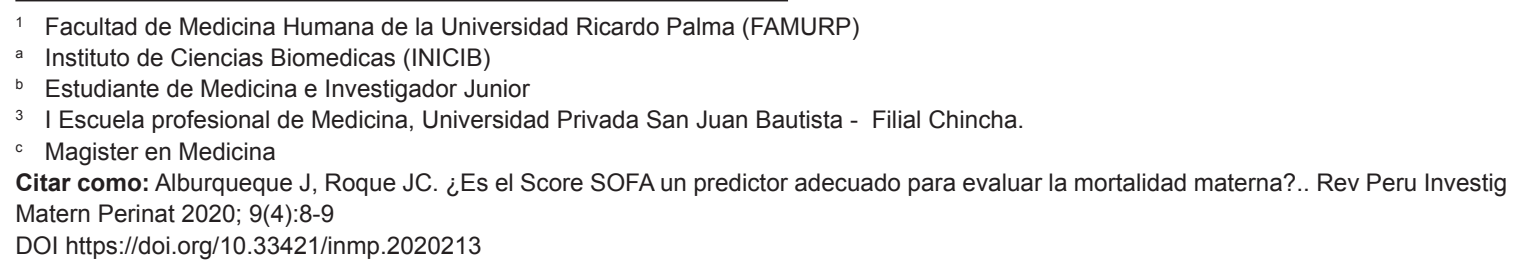


Declaración de conflictos de interés: los autores declaran no tener conflictos de interés en la publicación de este artículo.

\section{REFERENCIAS BIBLIOGRÁFICAS}

1. Jain S, Guleria K, Suneja A, Vaid NB, Ahuja S. Use of the Sequential Organ Failure Assessment score for evaluating outcome among obstetric patients admitted to the intensive care unit. International Journal of Gynecology \& Obstetrics. 2015;132(3): 332-336. doi:10.1016/j.jpo.2015.08.005

2. Dean Gopalan P, Muckart DJ. The critically ill obstetric patient: what's the score? International Journal of Obstetric Anesthesia. 2004;13(3):144-145. doi:10.1016/j. ijoa.2004.04.005

3. Bajwa SK, Bajwa SJ, Kaur J, Singh K, Kaur J. Is intensive care the only answer for high risk pregnancies in developing nations? J Emerg Trauma Shock. 2010;3(4):331-6. doi: 10.4103/0974-2700.70752
4. Lambden S, Laterre PF, Levy MM, Francois B. The SOFA score-development, utility and challenges of accurate assessment in clinical trials. Critical Care. 2019;23(1). doi:10.1186/s13054-019-2663-7

5. De Grooth HJ, Geenen IL, Girbes AR, Vincent JL, Parienti JJ, Oudemans-van Straaten HM. SOFA and mortality endpoints in randomized controlled trials: a systematic review and metaregression analysis. Critical Care.2017;21(1). doi:10.1186/ s13054-017-1609-1

\section{Correspondencia:}

Joseph Alburqueque Melgarejo

Dirección: Av. 1 de mayo 573 Carmen de la Legua, Reynoso

Correo: Joseph.a.m_3@hotmail.com

Teléfono: 979862474 